\title{
Multivariate thematic mapping using fractal glyphs
}

\author{
Antoni B. Moore ${ }^{\mathrm{a}, *}$, Bin Jiang ${ }^{\mathrm{b}}$ \\ ${ }^{a}$ National School of Surveying, University of Otago, Dunedin, New Zealand, tony.moore@otago.ac.nz \\ ${ }^{b}$ Faculty of Engineering and Sustainable development, Division of GIScience, University of Gävle, Gävle, Sweden, bin.jiang@hig.se \\ * Corresponding author
}

Keywords: Multivariate mapping, thematic cartography, fractal parameters

\begin{abstract}
:
Cartographers often face the task of depicting multivariate data on a single map. As spatial data gets ever more voluminous, and in particular, complex (multifarious), this challenge will have to be overcome with increasing regularity. Many solutions have been implemented in answer to situations like this: choropleths with trivariate colour or texture schemes, or point symbols in the form of star plots, ray glyphs or more naturalistically, Chernoff Faces (all of which could be sized proportional to a further variable).
\end{abstract}

Point symbols with a naturalistic basis have a mimetic appearance that makes for unambiguous communication of data. Chernoff faces in particular work so well due to their resemblance to, and human ability in reading, human faces. Importantly, facial features are easily linked with data through manipulation of their size, position or orientation (e.g. small to large nose; frowny to smily mouth).

Computer-generated fractals offer further potential solutions to the multivariate challenge in the naturalistic category. Examples such as the Barnsley fern leaf exhibit the fractal property of self-similar geometry over multiple spatial scales to create a realistic fern appearance. Fundamentally, the fern figure is under full control of a few numerical parameters that are simple to link with underlying attribute data. If these fractal glyphs are plotted as points on a map then we have a novel and potentially rich basis for multivariate mapping to address the big data challenge.

We can generate a Barnsley fern leaf fractal using the Iterated Function System (IFS), driven by parameters for geometric operators: displacement, rotation and scaling (Table 1). Each of these transforms can be linked with a variable, which is the basis for multivariate representation. Figure 1 illustrates how the appearance of the Barnsley fern is affected by scaling, overall orientation and angle of fern frond. In Table 1, cells $2 \mathrm{a}$ and $\mathrm{d}$ are used for scaling, $2 \mathrm{~b}$ for orientation and $3 \mathrm{~d} / 4 \mathrm{~d}$ for frond angle.

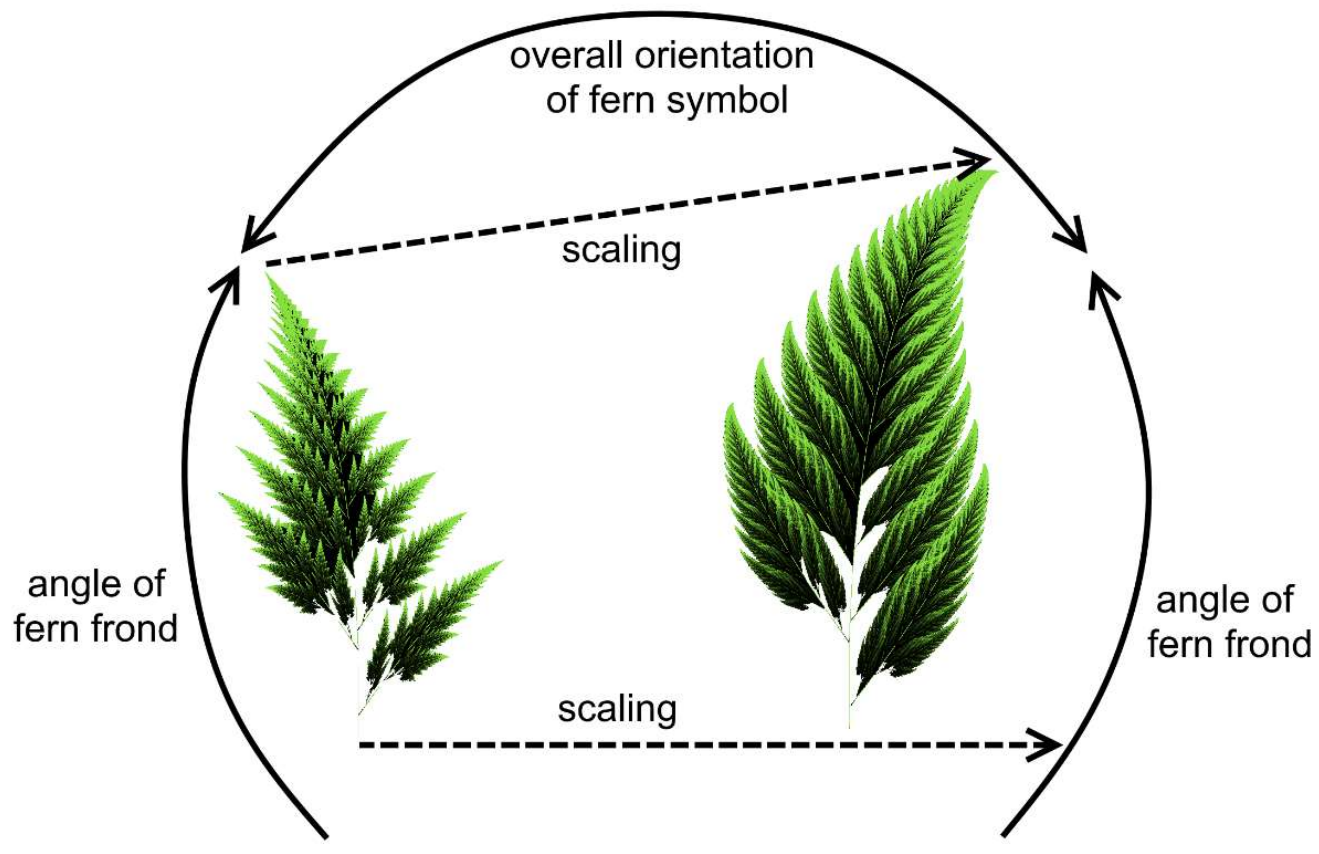

Figure 1. The Barnsley fern leaf as a multivariate symbol (Moore and Jiang, 2017). 


\begin{tabular}{r|rrrrrrr}
$\mathrm{W}$ & $\mathrm{a}$ & $\mathrm{b}$ & $\mathrm{c}$ & $\mathrm{d}$ & $\mathrm{e}$ & $\mathrm{f}$ & $\mathrm{p}$ \\
\hline 1 & 0.00 & 0.00 & 0.00 & 0.16 & 0.00 & 0.00 & 0.01 \\
2 & 0.85 & 0.04 & -0.04 & 0.85 & 0.00 & 1.60 & 0.85 \\
3 & 0.20 & -0.26 & 0.23 & 0.22 & 0.00 & 1.60 & 0.07 \\
4 & -0.15 & 0.28 & 0.26 & 0.24 & 0.00 & 0.44 & 0.07
\end{tabular}

Table 1. Fern IFS code in matrix format (Barnsley, 1993).

Our demonstrating example is based on "natural cities" (and within them, "natural streets" that define the city outline, Figure 2a), which have been calculated through the head/tail breaks method (Jiang, 2013) to capture a size-based emergent organic hierarchy. For natural streets, street network junction points have been triangulated, and triangular edges divided into two groups, those longer than the mean edge length (the "head") and those shorter (the "tail"). This process is recursively applied to the head, each time adding a tier to the street size hierarchy. Natural cities are calculated through a similar process, but on the basis of mean settlement area. In Figure $2 b$, natural city parameters have been used on the top 11 New Zealand cities in the following way: scaling has been linked to number of breaks (this is the ht-index, which indicates how complex the city network form is - Jiang and Yin, 2014), angle of fronds to percentage size of head and overall orientation to North (pointing right) and South Islands (left).

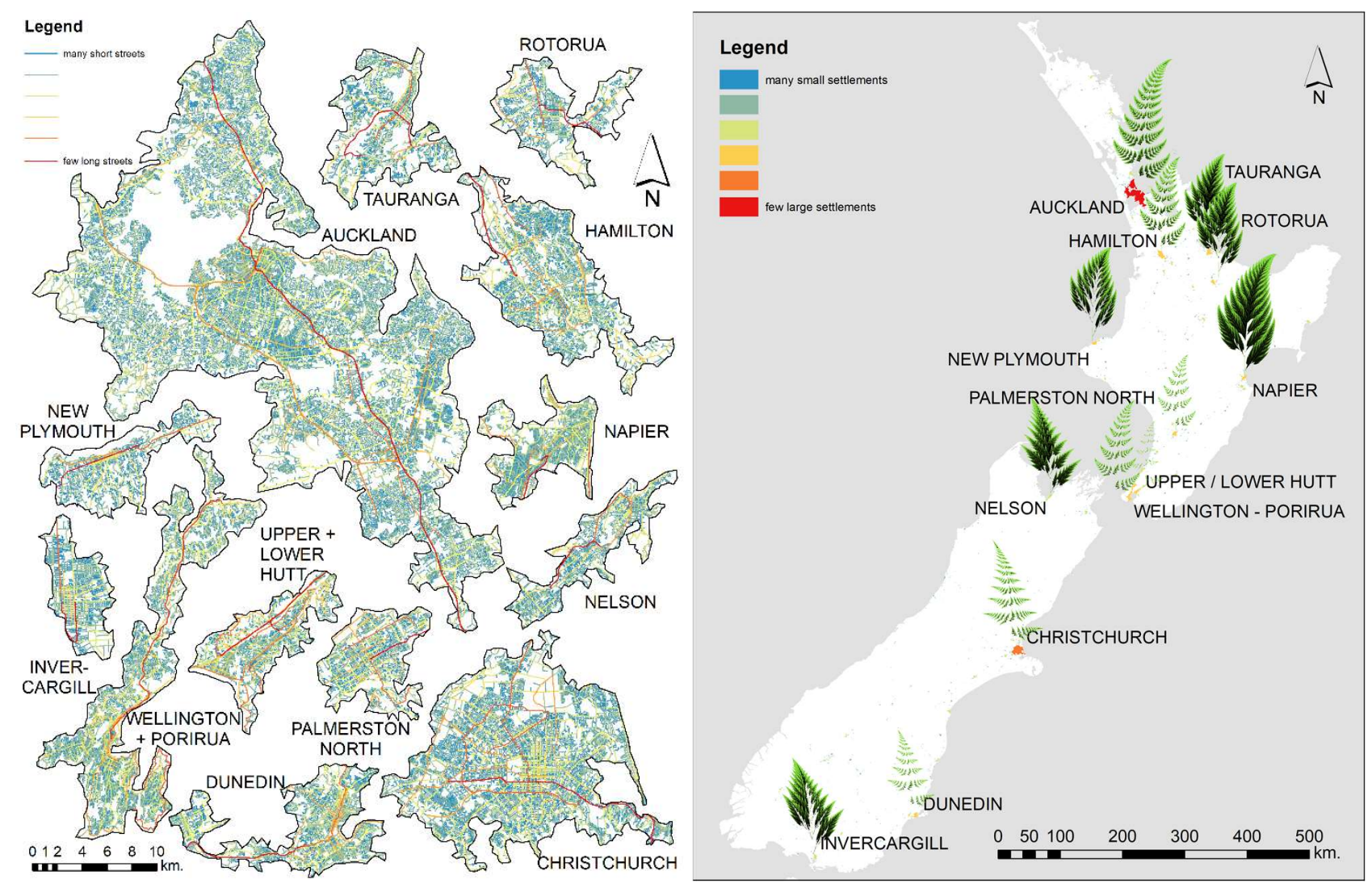

Figure 2. a) The outlines of the top 11 New Zealand natural cities, as defined by the contained natural streets; b) Map of New Zealand with fractal glyphs communicating "natural" settlement aspects of these cities

Rigorous testing needs to take place to measure the effect of fractal multivariate symbols compared with conventional cartographic means of representing the same data (which may include separate variable maps and the multivariate conventions listed above). Usability of fractally enhanced maps can be assessed along efficiency (how long it takes to perform a specific map task), effectiveness (how correct that task was performed) and satisfaction (covering ease, engagement and enjoyment of the map user in performing the map-based tasks).

Beyond multivariate mapping, these fractal glyphs are being investigated as one of the building blocks in generating data-based artworks (through the use of convolutional neural networks for style transfer - Moore and Jiang, 2017). This symbolisation based on self-similar graphics is an extension of a fractally-oriented vision for cartography (Jiang, 2018). It also addresses a part of the current cartography and Big Data agenda addressing spatial Big Data representation using artworks (Robinson et al, 2017). 